\title{
Applications of Sharpless asymmetric epoxidation in total synthesis
}

\author{
Majid M. Heravi ${ }^{1}$, Tahmineh BaieLashaki, Nasim Poorahmad \\ ${ }^{1}$ Department of Chemistry, Faculty of Physics \&Chemistry Alzahra University, Vanak, Tehran, Iran.
}

\begin{abstract}
This report presents the applications of enantioselective epoxidation of prochiral allylic alcohols, so called 'Sharpless asymmetric epoxidation', which is frequently referred as 'kinetic resolution'. This reaction results in the corresponding 2,3epoxy alcohols in high stereoselectivity as excellent starting materials for the synthesis of complex targets, such as naturally occurring biologically active molecules.
\end{abstract}

